Journal homepage http://revistas.unitru.edu.pe/index.php/SSMM

SELECCIONES MATEMÁTICAS
Universidad Nacional de Trujillo
ISSN: $2411-1783$ (Online)
Vol. 06(01): $108-118(2019)$

\title{
Weber's problem on the Riemannian Manifolds: Some upper bounds for the minimun Weber's function
}

\section{El problema de Weber sobre variedades Riemannianas: Algunas cotas Superiores para el mínimo de la función de Weber}

\author{
Franco Rubio López * , Patricia Edith Alvarez Rodriguez ** , Heyssen Dueñes Chávez ${ }^{* * *}$ \\ Received: Feb. 12, 2019 \\ Accepted: May. 19, 2019
}

DOI: http://dx.doi.org/10.17268/sel.mat.2019.01.13

\begin{abstract}
In this paper we obtain some upper bounds for the minimum of the Weber function on a strongly convex ball in a Riemannian manifold with positive sectional curvature; where the minimum is reached on the weighted geometric median of " $m$ " given points in the strongly convex.
\end{abstract}

Keywords: The Weber problem, Weighted Geometric Median, Riemannian manifold, Strongly convex set.

\section{Resumen}

En este artículo se obtiene algunas cotas superiores para el mínimo de la función de Weber sobre una bola fuertemente convexa en una variedad Riemanniana con curvartura seccional positiva; dicho mínimo se alcanza sobre la mediana geométrica pesada de "m" puntos dados en la bola fuertemente convexa.

Palabras Clave: Problema de Weber, Mediana Geométrica Pesada, Variedad Riemanniana, Conjunto fuertemente convexo.

\section{Introduction.}

Mathematically, Weber's problem in the plane consists in finding a point that minimizes the sum of the weighed distances to " $\mathrm{m}$ " fixed points. Weiszfeld [7] was the first to formulate an iterative method to approximate the solution to Weber's problem.

* ID ORCID: https://orcid.org/0000-0002-0168-3806, Departamento de Matemáticas, Universidad Nacional de Trujillo, Av. Juan Pablo II s/n, Ciudad Universitaria, Trujillo-Perú.(frubio@unitru.edu.pe)

* Departamento de Matemáticas, Universidad Nacional de Trujillo, Av. Juan Pablo II s/n, Ciudad Universitaria, TrujilloPerú (palvarezr@upao.edu.pe)

*** ID ORCID: https://orcid.org/0000-0001-5083-7800, Departamento de Matemáticas, Universidad Nacional de Trujillo, Av. Juan Pablo II s/n, Ciudad Universitaria, Trujillo-Perú.(ricardo18b@gmail.com)

This work is licensed under the Creative Commons Attribution-NoComercial-ShareAlike 4.0. 
If the fixed points are located in large regions on the surface of the earth, the approximation through a plane, and the use of the Euclidean distance, will provide unsatisfactory results; and since the sphere is used to model the planet earth, Drezner and Wesolowsky [2], Hansen [5], generalized Weber's problem to the sphere, and used the shortest arc distance to estimate the distance between two points.

Since both the plane and the sphere are regular surfaces; and since regular surfaces are a particular case of Riemannian manifolds, Fletcher [4], Aftab Khurrum [1], generalized the problem to Riemannian manifolds.

In addition, Fletcher [4] establishes conditions to guarantee the existence and uniqueness of the weighted geometric median. In this paper, upper bounds are established for the minimum of the Weber function on a strongly convex ball in a Riemannian manifolds; which is reached in the weighted geometric median.

\section{The Weber Problem on the Plane}

Consider " $m$ " different points in the plane $a_{1}, a_{2}, \ldots, a_{m} \in \mathbb{R}^{2}, a_{i}=\left(x_{i}, y_{i}\right), \forall i=1,2, \ldots, m$, with weights $w_{i}>0, \forall i=1,2, \ldots, m$.

Weber's problem is to find a point of $\mathbb{R}^{2}$ that minimizes the function $F: \mathbb{R}^{2} \rightarrow \mathbb{R}$, defined by:

$$
F(x, y)=\sum_{i=1}^{m} w_{i} \sqrt{\left(x-x_{i}\right)^{2}+\left(y-y_{i}\right)^{2}}
$$

ie:

$$
\min _{X \in \mathbb{R}^{2}} F(X)=\min _{X \in \mathbb{R}^{2}} \sum_{i=1}^{m} w_{i} d_{i}(X),
$$

where $d_{i}(X)=\sqrt{\left(x-x_{i}\right)^{2}+\left(y-y_{i}\right)^{2}}$ is the Euclidean distance between the point $X=(x, y)$ and the point $a_{i}=\left(x_{i}, y_{i}\right)$. The points $a_{i} \in \mathbb{R}^{2}$ are called vertices and the function $F(x)$ is called Weber function.

If the vertices $a_{i} \in \mathbb{R}^{2}$ are not collinear, the Weber function is strictly convex, so the problem (2) has a unique solution; Wendel and Hurter [8], proved that this solution is in the convex capsule of the vertices. Weiszfeld [7], proposed an iterative method to approximate the solution of the problem (2), by means of a sequence defined by:

$$
x^{k+1}=\frac{\sum_{i=1}^{m} \frac{w_{i} x_{i}}{d_{i}\left(x^{k}, y^{k}\right)}}{\sum_{i=1}^{m} \frac{w_{i}}{d_{i}\left(x^{k}, y^{k}\right)}} \quad, \quad y^{k+1}=\frac{\sum_{i=1}^{m} \frac{w_{i} y_{i}}{d_{i}\left(x^{k}, y^{k}\right)}}{\sum_{i=1}^{m} \frac{w_{i}}{d_{i}\left(x^{k}, y^{k}\right)}},
$$

with starting point:

$$
x^{0}=\frac{\sum_{i=1}^{m} w_{i} x_{i}}{\sum_{i=1}^{m} w_{i}} \quad, \quad y^{0}=\frac{\sum_{i=1}^{m} w_{i} y_{i}}{\sum_{i=1}^{m} w_{i}} .
$$

\section{The Weber Problem on Riemannian Manifold}

Let $\mathrm{M}$ be a Riemannian manifold $\mathrm{n}$-dimensional of class $C^{\infty}$, and $d_{M}, \nabla, \mathcal{R}$ are the intrinsic metric of M, Riemannian Connection and Curvature Operator respectively; where:

$$
\begin{aligned}
& \mathcal{R}(X, Y) Z=\nabla_{Y} \nabla_{X} Z-\nabla_{X} \nabla_{Y} Z+\nabla_{[X, Y]} Z, \quad \forall X, Y, Z \in \Gamma(M), \\
& \Gamma(M)=\left\{X: M \rightarrow T M / \quad X \in C^{\infty}(M)\right\}, \mathrm{TM} \text { is the tangent bundle of } \mathrm{M} .
\end{aligned}
$$


Definition 1. Let $S \subset M$. S is called a Strongly Convex set if for each pair of points $q_{1}, q_{2}$ of the closed set $\bar{S}$ of $S$, there is a unique minimizing geodesic $\alpha:[0,1] \rightarrow S, \alpha(0)=q_{1}, \alpha(1)=q_{2}$, such that $\alpha([0,1]) \subset S$.

Also, for each $p \in M$, there is $\delta>0$, such that the geodesic ball $B(p, \delta)$ is strongly convex [6]. Let $A \subset M$ be a strongly convex set in $M$. The set $T_{x} A$ of tangent vectors to $A$ at point $x$ is a convex cone in tangent space to $M$ at $x$ : $T_{x} M$.

Given $X_{x}, Y_{x} \in T_{x} A$, then: $t X_{x}+t Y_{x} \in T_{x} A, \forall t \geq 0$; and there are curves:

$$
\alpha, \beta:[0,1] \rightarrow A, \alpha(0)=\beta(0)=x, \alpha^{\prime}(0)=X_{x} \quad, \beta^{\prime}(0)=Y_{x} .
$$

Also, we consider a deformation of class $C^{\infty}$ of curves in $A$ :

$$
\begin{gathered}
\tau:[0,1] x\left[0, t_{0}\right] \rightarrow A \\
(s, t) \rightarrow \tau(s, t)
\end{gathered}
$$

such that

a) $\tau(s, 0)=x$.

b) $\tau(0, t)=\alpha(t)=\exp _{x}\left(t X_{x}\right)$,

$$
\tau(1, t)=\beta(t)=\exp _{x}\left(t Y_{x}\right)
$$

c) For each $t \in\left[0, t_{0}\right]$, the curve:

$$
\gamma_{t}:[0,1] \rightarrow A \text {, defined by } \gamma_{t}(s)=\tau(s, t),
$$

is a parameterized geodesic with a parameter proportional to the arc length. This allows to consider the parameterized geodesic:

$$
\lambda_{s}:\left[0, t_{0}\right] \rightarrow A, \forall s \in[0,1] \text {, such that } \lambda_{s}(t)=\gamma_{t}(s)=\tau(s, t) .
$$

Theorem 1. The vector field $J(s)=\frac{\partial \tau(s, t)}{\partial t}, \forall s \in[0,1]$, defined along the curve $\gamma_{t}(s)=\tau(s, t)$, is a field of Jacobi; ie:

$$
\frac{D^{2} J(s)}{\partial s^{2}}+\mathcal{R}\left(\frac{d \gamma_{t}(s)}{d s}, J(s)\right) \frac{d \gamma_{t}(s)}{d s}=0
$$

Proof

Using the curvature operator $\mathcal{R}$, we have:

$$
\begin{aligned}
\mathcal{R}\left(\frac{d \gamma_{t}(s)}{d s}, J(s)\right) \frac{d \gamma_{t}(s)}{d s} & =\nabla_{J(s)} \nabla_{\frac{d \gamma t(s)}{d s}} \frac{d \gamma_{t}(s)}{d s}-\nabla_{\frac{d t_{t}(s)}{d s} \nabla_{J(s)}} \frac{d \gamma_{t}(s)}{d s}+\nabla_{\left[\frac{d \gamma_{t}(s)}{d s}, J(s)\right]} \frac{d \gamma_{t}(s)}{d s} \\
& =-\nabla_{\frac{d \gamma_{t}(s)}{d s}} \nabla_{\nabla_{\frac{d t_{t}(s)}{d t}}} \frac{d \lambda_{s}(t)}{d t}=-\frac{D^{2} J(s)}{\partial s^{2}} .
\end{aligned}
$$

Therefore, $J(s)$ is a field of Jacobi. 
Theorem 2. Let $\tau(s, t)$ be a deformation given by (4), and be the geodesic:

$$
\lambda_{s}:\left[0, t_{0}\right] \rightarrow A, \forall s \in[0,1] \text {, such that } \lambda_{s}(t)=\gamma_{t}(s)=\tau(s, t) .
$$

Then:

$$
\frac{d \lambda_{s}(0)}{d t}=(1-s) X_{x_{0}}+s Y_{x_{0}}, \forall s \in[0,1]
$$

\section{Proof}

By theorem (1), the vector field $J(s)=\frac{\partial \tau(s, t)}{\partial t}, \forall s \in[0,1]$, is a field of Jacobi along the geodesic $\gamma_{t}(s)=\tau(s, t)$.

In particular for $t=0$, and by (a) of the deformation (4), the Jacobi equation is reduced:

$$
\frac{D^{2} J(s)}{\partial s^{2}}=\frac{D}{d s} \frac{D}{d s}(J(s))=\frac{D}{d s} \frac{D}{d s}\left(\frac{\partial \tau(s, 0)}{\partial t}\right)=0 .
$$

By the conditions:

$$
\frac{\partial \tau(0,0)}{\partial t}=\frac{d \alpha(0)}{d t}=X_{x_{0}}, \quad \frac{\partial \tau(1,0)}{\partial t}=\frac{d \beta(0)}{d t}=Y_{x_{0}}
$$

we have:

$$
\frac{d \lambda_{s}(0)}{d t}=(1-s) X_{x_{0}}+s Y_{x_{0}}, \forall s \in[0,1] .
$$

Definition 2. Given points $x_{1}, x_{2}, \ldots, x_{m} \in M$, and positive real number $w_{1}, w_{2}, \ldots, w_{m}$, the function $F: M \rightarrow \mathbb{R}$, defined by:

$$
F(x)=\sum_{i=1}^{m} w_{i} d_{M}\left(x, x_{i}\right)
$$

is called Weber's function.

Theorem 3. Let $p_{0} \in M, B\left(p_{0}, r\right)$ a strongly convex ball. For each $x_{0} \in B\left(p_{0}, r\right)$, the function $F_{x_{0}}: B\left(p_{0}, r\right) \rightarrow \mathbb{R}$ defined by $F_{x_{0}}(x)=d_{M}\left(x_{0}, x\right)$ is a convex function.

\section{Proof}

Let $x_{1}, x_{2} \in B\left(p_{0}, r\right)$ be two arbitrary points. Then there are minimal geodesics

$$
\begin{gathered}
\alpha:[0,1] \rightarrow B\left(p_{0}, r\right), \alpha(0)=x_{0}, \alpha(1)=x_{1}, \frac{d \alpha(0)}{d t}=X_{x_{0}} \\
\beta:[0,1] \rightarrow B\left(p_{0}, r\right), \beta(0)=x_{0}, \beta(1)=x_{2}, \frac{d \beta(0)}{d t}=Y_{x_{0}},
\end{gathered}
$$

such that:

$$
\begin{aligned}
& l(\alpha)=\int_{0}^{1}\left\|\frac{d \alpha(t)}{d t}\right\| d t=\left\|X_{x_{0}}\right\|, \\
& l(\beta)=\int_{0}^{1}\left\|\frac{d \beta(t)}{d t}\right\| d t=\left\|Y_{x_{0}}\right\| .
\end{aligned}
$$

By deformation $\tau(s, t)$ given by (4), there is a minimal geodesic: $\gamma_{1}:[0,1] \rightarrow B\left(p_{0}, r\right)$, such that:

$$
\begin{aligned}
& \gamma_{1}(0)=\tau(0,1)=\alpha(1)=x_{1}, \\
& \gamma_{1}(1)=\tau(1,1)=\beta(1)=x_{2} .
\end{aligned}
$$


For each "s" consider the geodesic:

$$
\lambda_{s}:[0,1] \rightarrow B\left(p_{0}, r\right), \quad \lambda_{s}(t)=\tau(s, t)
$$

what satisfies: $\lambda_{s}(0)=\tau(s, 0)=x_{0}, \quad \lambda_{s}(1)=\tau(s, 1)=\gamma_{1}(s)$.

Then, the function $F_{x_{0}} \diamond \gamma_{1}:[0,1] \rightarrow \mathbb{R}$ verifies:

$$
\begin{aligned}
\left(F_{x_{0}}\right. & \left.\diamond \gamma_{1}\right)(s)=F_{x_{0}}\left(\gamma_{1}(s)\right)=F_{x_{0}}\left(\lambda_{s}(1)\right)=d_{M}\left(x_{0}, \lambda_{s}(1)\right)= \\
\quad & =\int_{0}^{1}\left\|\frac{d \lambda_{s}(t)}{d t}\right\| d t=\left\|\frac{d \lambda_{s}(0)}{d t}\right\|=\left\|(1-s) X_{x_{0}}+s Y_{x_{0}}\right\| \\
& \leq(1-s)\left\|X_{x_{0}}\right\|+s\left\|Y_{x_{0}}\right\| \\
& =(1-s) d_{M}\left(x_{0}, \alpha(1)\right)+s d_{M}\left(x_{0}, \beta(1)\right) \\
& =(1-s) d_{M}\left(x_{0}, x_{1}\right)+s d_{M}\left(x_{0}, x_{2}\right) \\
& =(1-s) F_{x_{0}}\left(x_{1}\right)+s F_{x_{0}}\left(x_{2}\right), \forall s \in[0,1]
\end{aligned}
$$

Therefore, $F_{x_{0}}$ is a convex function.

Theorem 4. Let $x_{1}, x_{2}, \ldots, x_{m} \in B\left(p_{0}, r\right)$. Then the function $F: B\left(p_{0}, r\right) \rightarrow \mathbb{R}$ defined by:

$$
F(x)=\sum_{i=1}^{m} w_{i} d_{M}\left(x, x_{i}\right), w_{i}>0, \forall i=1, \ldots, m
$$

is convex function.

\section{Proof}

By theorem (3), the Weber's function (6):

$$
\begin{aligned}
F(x) & =\sum_{i=1}^{m} w_{i} d_{M}\left(x, x_{i}\right) \\
& =\sum_{i=1}^{m} w_{i} F_{x_{i}}(x)
\end{aligned}
$$

where the functions $F_{x_{i}}, \forall i=1, \ldots, m$, are convex functions. Also,

$$
w_{i} F_{x_{i}}, \forall i=1, \ldots, m, \text { are convex functions. }
$$

Therefore: $F(x)=\sum_{i=1}^{m} w_{i} d_{M}\left(x, x_{i}\right)$ is a convex function.

\section{Some bound for the Weber function}

In this section we introduce the weighted geometric median (Fletcher, 2009).

Definition 3. Let $U \subset M$ be a strongly convex set, $x_{1}, x_{2}, \ldots, x_{m} \in U$. The Weighted Geometric Median of $F(x)=\sum_{i=1}^{m} w_{i} d_{M}\left(x, x_{i}\right), \quad w_{i}>0, \forall i=1, \ldots, m, \quad \sum_{i=1}^{m} w_{i}=1$, is:

$$
x^{*}=\arg \min _{x \in U} \sum_{i=1}^{m} w_{i} d_{M}\left(x, x_{i}\right)
$$

If $w_{i}=\frac{1}{m}, \forall i=1, \ldots, m, x^{*}$ is call simply the Geometric Median [4]. 
Let $p_{0} \in M, B\left(p_{0}, r\right)=\exp _{p_{0}}(B(0, r)), B(0, r) \subset T_{p_{0}} M$ is the ball open in tangent space $T_{p_{0}} M$, where the exponential map $\exp _{p_{0}}$ is a diffeomorphism.

Now, let's consider the points $x_{1}, x_{2}, \ldots, x_{m} \in B\left(p_{0}, r\right)$, and be

$$
v_{i}=\exp _{p_{0}}^{-1}\left(x_{i}\right) \in B(0, r), \forall i=1, \ldots, m \text {. }
$$

Theorem 5. Let $\mathrm{M}$ be a Riemannian manifold with sectional curvature $k(x)>0, \forall x \in M$. Then:

$$
F(x)=\sum_{i=1}^{m} w_{i} d_{M}\left(x, x_{i}\right)<2 r .
$$

Proof

As $k(x)>0, \forall x \in M$, and by Toponogov's theorem, we have:

Also:

$$
\begin{aligned}
& d_{M}\left(x, x_{i}\right) \leq\left\|v-v_{i}\right\|, \quad \forall i=1, \ldots, m \\
& d_{M}\left(x, x_{i}\right) \leq\left\|v-v_{i}\right\|<2 r, \quad \forall i=1, \ldots, m
\end{aligned}
$$$$
w_{i} d_{M}\left(x, x_{i}\right) \leq w_{i}\left\|v-v_{i}\right\|<2 r w_{i}, \quad \forall i=1, \ldots, m
$$$$
\sum_{i=1}^{m} w_{i} d_{M}\left(x, x_{i}\right) \leq \sum_{i=1}^{m} w_{i}\left\|v-v_{i}\right\|<2 r \sum_{i=}^{m} w_{i}=2 r
$$

Therefore:

$$
F(x)=\sum_{i=1}^{m} w_{i} d_{M}\left(x, x_{i}\right)<2 r .
$$

Theorem 6. Let $\mathrm{M}$ be a Riemannian manifold with sectional curvature $0<k(x) \leq \Delta$, $B\left(p_{0}, r\right) \subset M$ a strongly convex ball, $r<\frac{\pi}{4 \sqrt{\Delta}}$, and $x_{1}, x_{2}, \ldots, x_{m} \in B\left(p_{0}, r\right)$, then exists and is unique the weighted gemetric median defined by (8). Also:

$$
F(x)=\sum_{i=1}^{m} w_{i} d_{M}\left(x, x_{i}\right)<\frac{\pi}{2 \sqrt{\Delta}} .
$$

\section{Proof}

By theorem (4) the function $F(x)=\sum_{i=1}^{m} w_{i} d_{M}\left(x, x_{i}\right)$ is convex; and how the sectional curvature of $M$ is bounded, and $r<\frac{\pi}{4 \sqrt{\Delta}}$, then by Fletcher [4], exists and is unique the weighted gemetric median defined by (8).

By theorem (5):

$$
F(x)=\sum_{i=1}^{m} w_{i} d_{M}\left(x, x_{i}\right)<2 r<\frac{\pi}{2 \sqrt{\Delta}} .
$$

Therefore:

$$
F(x)=\sum_{i=1}^{m} w_{i} d_{M}\left(x, x_{i}\right)<\frac{\pi}{2 \sqrt{\Delta}} .
$$

Corollary 1. Let $\mathrm{M}$ be a Riemannian manifold with sectional curvature $0<k(x) \leq \Delta$, $B\left(p_{0}, r\right) \subset M$ a strongly convex ball, $r<\frac{\pi}{4 \sqrt{\Delta}}$, and $x_{1}, x_{2}, \ldots, x_{m} \in B\left(p_{0}, r\right)$, then

$$
F^{*}=\min _{x \in B\left(p_{0}, r\right)} \sum_{i=1}^{m} w_{i} d_{M}\left(x, x_{i}\right)<\frac{\pi}{2 \sqrt{\Delta}} .
$$


By theorem (6), exists and is unique the weighted geometric median $x^{*} \in B\left(p_{0}, r\right)$; and be $F^{*}=F\left(x^{*}\right)$. Then:

$$
F^{*}=F\left(x^{*}\right) \leq F(x)<\frac{\pi}{2 \sqrt{\Delta}}, \forall x \in B\left(p_{0}, r\right) .
$$

Let $x_{1}, x_{2}, \ldots, x_{m} \in B\left(p_{0}, r\right), \quad v_{i}=\exp _{p_{0}}^{-1}\left(x_{i}\right) \in B(0, r), \forall i=1, \ldots, m$,

$$
v=\exp _{p_{0}}^{-1}(x) \in B(0, r), \forall x \in B\left(p_{0}, r\right) .
$$

This allows to consider the function $f: B(0, r) \subset T_{p_{0}} M \rightarrow \mathbb{R}$, defined by:

$$
f(v)=\sum_{i=1}^{m} w_{i}\left\|v-v_{i}\right\| .
$$

Theorem 7. Let $\mathrm{M}$ be a Riemannian manifold with sectional curvature $k(x)>0, \forall x \in M$. Then:

$$
F(x) \leq f(v), \quad \forall x \in B\left(p_{0}, r\right) .
$$

\section{Proof}

As $k(x)>0, \forall x \in M$, and by Toponogov theorem we have:

$$
d_{M}\left(x, x_{i}\right) \leq\left\|v-v_{i}\right\|, \forall i=1, \ldots, m .
$$

Also:

$$
w_{i} d_{M}\left(x, x_{i}\right) \leq w_{i}\left\|v-v_{i}\right\|, \quad w_{i}>0, \forall i=1, \ldots, m .
$$

Then

$$
F(x)=\sum_{i=1}^{m} w_{i} d_{M}\left(x, x_{i}\right) \leq \sum_{i=1}^{m} w_{i}\left\|v-v_{i}\right\|=f(v) .
$$

Therefore:

$$
F(x) \leq f(v), \quad \forall x \in B\left(p_{0}, r\right)
$$

Theorem 8. Let $\mathrm{M}$ be a Riemannian manifold with sectional curvature $0<k(x) \leq \Delta$, $B\left(p_{0}, r\right) \subset M$ a strongly convex ball, $r<\frac{\pi}{4 \sqrt{\Delta}}$, and be a $x_{1}, x_{2}, \ldots, x_{m} \in B\left(p_{0}, r\right)$,

$$
\sum_{i=1}^{m} w_{i}=1, \quad F^{*}=\min _{x \in B\left(p_{0}, r\right)}(F(x)), \quad f^{*}=\min _{v \in B(0, r)}(f(v)) .
$$

Then:

$$
F^{*} \leq f^{*}<\frac{\pi}{2 \sqrt{\Delta}}
$$

\section{Proof}

Let $x^{*} \in B\left(p_{0}, r\right)$ such that: $F^{*}=F\left(x^{*}\right)$, and $v^{*} \in B(0, r)$ such that: $f^{*}=f\left(v^{*}\right)$. We have

Case 1. Let $v^{*}=\exp _{p_{0}}^{-1}\left(x^{*}\right)$, then:

$$
F^{*}=F\left(x^{*}\right) \leq f\left(v^{*}\right)=f^{*}<\frac{\pi}{2 \sqrt{\Delta}} .
$$

Case 2. Let $v_{x^{*}}=\exp _{p_{0}}^{-1}\left(x^{*}\right)$, such that $v_{x^{*}} \neq v^{*}$, then $f\left(v^{*}\right)<f\left(v_{x^{*}}\right)$.

As $F(x) \leq f(v)$, y $v=\varphi(x)$, where $\varphi=\exp _{p_{0}}^{-1}$, we have: 


$$
F(x) \leq f(\varphi(x))=G(x), \text { where } G=f \circ \varphi: B\left(p_{0}, r\right) \rightarrow \mathbb{R}
$$

then:

$$
\begin{aligned}
\min _{x \in B\left(p_{0}, r\right)}(F(x)) \leq & \min _{x \in B\left(p_{0}, r\right)}(G(x)) \\
& =\min _{x \in B\left(p_{0}, r\right)}(f \circ \varphi(x)) \\
& =f^{*}=\min _{x \in B\left(p_{0}, r\right)}(f(v))<\frac{\pi}{2 \sqrt{\Delta}} .
\end{aligned}
$$

Therefore:

$$
F^{*} \leq f^{*}<\frac{\pi}{2 \sqrt{\Delta}}
$$

\section{Example}

In this section we present results on the unit sphere.

Let $M=S^{2}=\left\{(x, y, z) \in \mathbb{R}^{3} / x^{2}+y^{2}+z^{2}=1\right\}$ the unit sphere, whose sectional curvature $k(x)=1, \forall x \in S^{2}$; therefore the bounded above for the sectional curvature is $\Delta=1$.

Now, consider the ball strongly convex $B\left(p_{0}, r\right)$, where $p_{0}=(0,0,1)$, and $r=\frac{\pi}{4}$, Drezner and Wesolowsky [2], and using spherical Coordinates:

$$
x=\cos (\varphi) \cos (\theta), \quad y=\cos (\varphi) \sin (\theta), \quad z=\sin (\varphi), \quad \frac{\pi}{4}<\varphi<\frac{\pi}{2}, 0<\theta<2 \pi,
$$

a PC with Intel Core i3 microprocessor, Windows 7 operating system, $2.20 \mathrm{GHz}, 4 \mathrm{~GB}$ of RAM,

\begin{tabular}{|c|c|}
\hline \multicolumn{2}{|r|}{ Results } \\
\hline Optimal Value: $\quad F^{*}$ & 0.3222718 \\
\hline Geometric Median: $x^{*}$ & $(0.0436559,-0.0280022,0.9986541)$ \\
\hline
\end{tabular}
we have the following cases:

Case 1. We consider $m=5$ points chosen randomly

\begin{tabular}{|c|c|c|c|}
\hline \multicolumn{4}{|c|}{ Coordinates and weights } \\
\hline & $\theta_{i}$ & $\varphi_{i}$ & $w_{i}$ \\
\hline 1 & 5.1191 & 0.8620 & 0.2000 \\
\hline 2 & 5.6913 & 1.0041 & 0.2000 \\
\hline 3 & 0.7979 & 1.2149 & 0.2000 \\
\hline 4 & 5.7389 & 1.5374 & 0.2000 \\
\hline 5 & 3.9732 & 1.5432 & 0.2000 \\
\hline
\end{tabular}

\begin{tabular}{|c|c|}
\hline \multicolumn{2}{|r|}{ Results } \\
\hline Optimal Value: & 0.3365514 \\
\hline Geometric Median: $v^{*}$ & $(0.0445683,-0.0286065,1.0000000)$ \\
\hline
\end{tabular}

Table 1. Spherical coordinates of the points and their weights.

Using the Weiszfeld algorithm in the sphere was obtained:

Table 2. The Geometric Median and the Optimal value on the Sphere.

Using the Weiszfeld algorithm in the tangent space $T_{p_{0}} S^{2}$ was obtained:

Table 3. The Geometric Median and the Optimal value on the Tangent space $T_{p_{0}} S^{2}$. 
Table 2 and Table 3 show that: $F^{*}<f^{*}$.

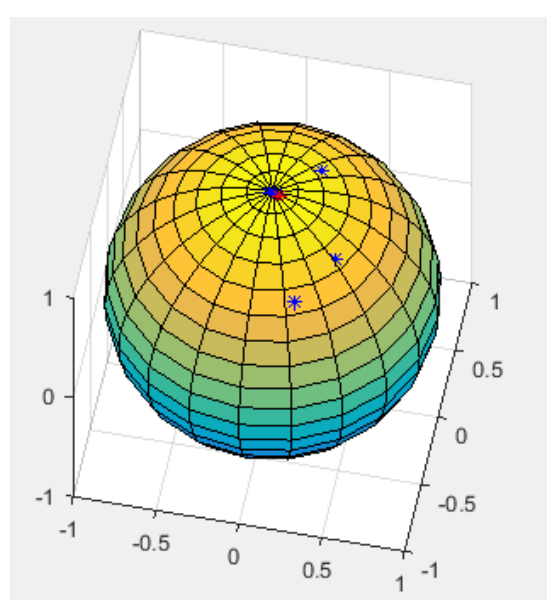

Figure 1. The red point is the geometric median for 5 points.

Case 2. We consider $m=10$ points chosen randomly

\begin{tabular}{|c|c|c|c|}
\hline \multicolumn{4}{|c|}{ Coordinates and weights } \\
\hline & $\theta_{i}$ & $\varphi_{i}$ & $w_{i}$ \\
\hline 1 & 4.4362 & 1.1300 & 0.1000 \\
\hline 2 & 0.2000 & 1.0851 & 0.1000 \\
\hline 3 & 1.7400 & 1.3866 & 0.1000 \\
\hline 4 & 0.2901 & 1.4099 & 0.1000 \\
\hline 5 & 0.6103 & 0.9322 & 0.1000 \\
\hline 6 & 5.1739 & 1.1701 & 0.1000 \\
\hline 7 & 4.3657 & 1.1354 & 0.1000 \\
\hline 8 & 1.9924 & 1.2930 & 0.1000 \\
\hline 9 & 5.9704 & 1.3425 & 0.1000 \\
\hline 10 & 0.2164 & 1.3781 & 0.1000 \\
\hline
\end{tabular}

Table 4. Spherical coordinates of the points and their weights.

Using the Weiszfeld algorithm in the sphere was obtained:

\begin{tabular}{|lc|cc|}
\hline \multicolumn{3}{|c|}{ Results } \\
\hline Optimal Value: & $F^{*}$ & 0.3029679 \\
\hline Geometric Median: $x^{*}$ & $(0.1638406$, & 0.0266638, & $0.9861264)$ \\
\hline
\end{tabular}

Table 5. The Geometric Median and the Optimal value on the Sphere

Using the Weiszfeld algorithm in the tangent space $T_{p_{0}} S^{2}$ was obtained:

\begin{tabular}{|c|c|}
\hline \multicolumn{2}{|r|}{ Results } \\
\hline Optimal Value: & 0.3134207 \\
\hline Geometric Median: $v$ & $(0.1633452, \quad 0.0262530,1.0000000)$ \\
\hline
\end{tabular}

Table 6. The Geometric Median and the Optimal value on the Tangent space $T_{p_{0}} S^{2}$.

Table 5 and Table 6 show that: $F^{*}<f^{*}$. 


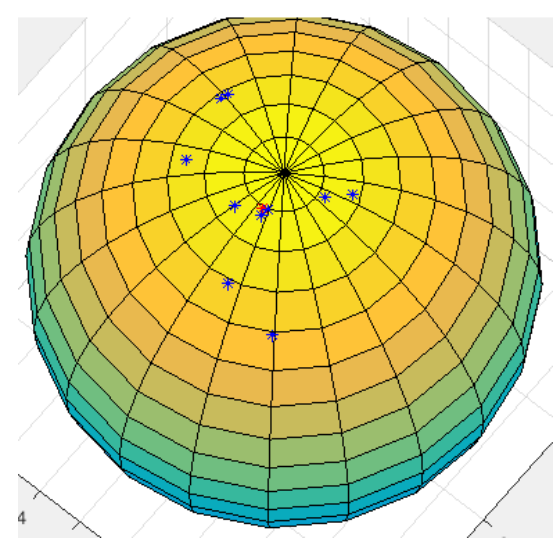

Figure 2. The red point is the geometric median for 10 points.

Case 3. We consider $m=20$ points chosen randomly

\begin{tabular}{|c|c|c|c|}
\hline \multicolumn{4}{|c|}{ Coordinates and weights } \\
\hline & $\theta_{i}$ & $\varphi_{i}$ & $w_{i}$ \\
\hline 1 & 1.3053 & 1.2526 & 0.0500 \\
\hline 2 & 1.8928 & 0.9913 & 0.0500 \\
\hline 3 & 2.9589 & 1.2589 & 0.0500 \\
\hline 4 & 1.4482 & 1.3440 & 0.0500 \\
\hline 5 & 5.3049 & 0.9596 & 0.0500 \\
\hline 6 & 1.2237 & 0.8776 & 0.0500 \\
\hline 7 & 1.4195 & 1.0184 & 0.0500 \\
\hline 8 & 1.0726 & 1.0358 & 0.0500 \\
\hline 9 & 1.4305 & 1.1185 & 0.0500 \\
\hline 10 & 2.7376 & 1.1843 & 0.0500 \\
\hline 11 & 1.9547 & 0.8526 & 0.0500 \\
\hline 12 & 5.8018 & 0.9916 & 0.0500 \\
\hline 13 & 2.7031 & 1.4145 & 0.0500 \\
\hline 14 & 1.1612 & 0.8083 & 0.0500 \\
\hline 15 & 5.6855 & 1.5149 & 0.0500 \\
\hline 16 & 6.1559 & 1.3590 & 0.0500 \\
\hline 17 & 2.7575 & 1.1692 & 0.0500 \\
\hline 18 & 0.6982 & 1.2398 & 0.0500 \\
\hline 19 & 1.6215 & 0.9718 & 0.0500 \\
\hline 20 & 2.5681 & 1.1458 & 0.0500 \\
\hline
\end{tabular}

Table 7. Spherical coordinates of the points and their weights.

Using the Weiszfeld algorithm in the sphere was obtained:

\begin{tabular}{|c|c|}
\hline \multicolumn{2}{|r|}{ Results } \\
\hline Optimal Value: $\quad F^{*}$ & 0.3630473 \\
\hline Geometric Median: $x^{*}$ & $(0.0300179,0.2946810,0.9551241)$ \\
\hline
\end{tabular}

Table 8. The Geometric Median and the Optimal value on the Sphere.

Using the Weiszfeld algorithm in the tangent space $T_{p_{0}} S^{2}$ was obtained:

\begin{tabular}{|l|c|}
\hline \multicolumn{3}{|c|}{ Results } \\
\hline Optimal Value: $\quad f^{*}$ & 0.3653496 \\
\hline Geometric Median: $v^{*}$ & $(0.0280437,0.2905243,1.0000000)$ \\
\hline
\end{tabular}

Table 9. The Geometric Median and the Optimal value on the Tangent space $T_{p_{0}} S^{2}$. 
Table 8 and Table 9 show that: $F^{*}<f^{*}$.

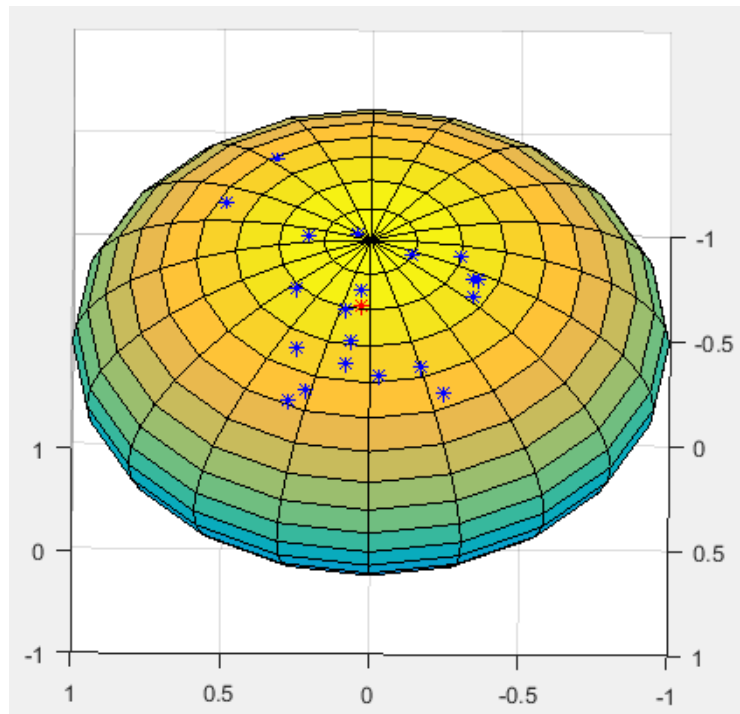

Figure 3 . The red point is the geometric median for 20 points.

\section{References}

[1]. Aftab, K., Hartley, R., and Trumpf, J. Generalized Weiszfeld Algorithms for Lq Optimization. IEEE Transactions on Pattern Analysis and Machine Intelligence, 37(4), 728745, 2015. doi:10.1109/tpami.2014.2353625

[2]. Drezner, W and Wesolowsky, G.O. Facility Location on the Sphere. Journal of the Operational Research Society, 29, 997-1004, 1978.

[3]. Drezner, W. A Solution to the Weber Location Problem on the Sphere. Journal of the Operational Research Society, 36, 333-338, 1985.

[4]. Fletcher, T; Venkatasubramanian, $\mathrm{V}$ and Joshi, S. The geometric median on Riemannian manifolds with application to robust atlas estimation. Neurolmage 45, s143-s152, 2009.

[5]. Hansen, P; Jaumard, B and Krau, S. A algorithm for Weber's Problem on the Sphere. Location Science 3(4), 217-237, 1995.

[6]. P. Do Carmo, M. Geometria Riemanniana. IMPA, Rio de Janeiro, 1979.

[7]. Weiszfeld, E. V. Sur le point pour lequel la Somme des distances de $\mathrm{n}$ point donnés est minisum. The Tohoku Mathematical Journal, 43, 335-386, 1937.

[8]. Wendel, R and Hurter, A. Location Theory, dominance and convexity. Operations Research, 21(1), 314-320, 1973.

[9]. Wesolowsky, G.O. Location Problem on a Sphere. Regional Science and Urban Economics, 12, 495-508, 1982. 